\title{
Wissensgeschichtliche Perspektiven auf das Militär: Eine Einleitung*
}

\author{
Pascal Germann, Heinrich Hartmann
}

Militär und Wissenschaft bilden zwei Systeme, die auf den ersten Blick wenig miteinander zu tun haben, zumal sie Werte verkörperten und verkörpern, die unterschiedlicher nicht sein könnten. Werden Wissenschaften mit dem Ideal des kritischen Geistes, des zwanglosen Arguments und des freien Austausches verbunden, erscheint das Militär als Ort, in welchem traditionelle Tugenden wie Gehorsam, Unterordnung und Disziplin eine erstaunliche Beständigkeit erlebten. Während die Wissenschaft im 19. Jahrhundert zur Domäne des aufstrebenden Bürgertums avancierte, die ihren Siegeszug mit dem Versprechen von Fortschritt und Modernität antrat, hielt das Militär wie der Historiker Dan Diner betont - lange Zeit an «adeligen Werten und Lebensformen» fest und übte gerade als «Aufhalter des anbrandenden Fortschritts» eine gesellschaftliche Attraktivität aus. ${ }^{1}$ Im 20. Jahrhundert wurde verschiedentlich ein Zusammenhang von Wissenschaft und Demokratie postuliert; 2 demgegenüber führten die Erfahrungen dieses Jahrhunderts dazu, dass ein mächtiges Militär oder eine 'militarisierte' Gesellschaft gemeinhin als Indizien für die Abwesenheit oder ein Scheitern von Demokratie gewertet werden. Wird der Siegeszug der Wissenschaften in der Regel mit den Segnungen und Wohltaten der Moderne assoziiert, gilt das Militär nicht selten als jene Institution, die wie kaum eine andere mit der destruktiven und gewalttätigen Kehrseite der Moderne in Verbindung gebracht wird.

Zum Verhältnis von Wissenschaft und Militär existiert indessen auch ein entgegengesetztes Bild, das ebenfalls eine breite Wirkung entfaltete und mit Vorliebe zur Apologie von Kriegen bemüht wurde. Gemeint ist die Behauptung, dass Krieg nicht nur Zerstörung und Leid mit sich bringe, sondern auch

* Wir danken Jakob Vogel und zwei anonymen Gutachtern für die Kommentare und Anmerkungen zur Konzeption dieses Heftes und seiner einzelnen Beiträge.

1 Diner 2000, 39.

2 Siehe dazu Hagner 2012. 
Gutes bewirke, indem er den wissenschaftlichen, medizinischen und technischen Fortschritt befördere. Mit einem aus seinem ursprünglichen Sinnzusammenhang gerissenen Herakles-Zitat, das den Krieg als «Vater aller Dinge» bezeichnet, ließ sich die Überzeugung auf eine einfache und einprägsame Formel bringen. ${ }^{3}$ Das Militär wäre demnach nicht ein antiaufklärerischer Gegenpart zur Welt der Wissenschaft, sondern würde zumindest im Ernstfall als Förderer der wissenschaftlichen Entwicklung fungieren. Auch innerhalb dieser Vorstellung bleiben jedoch Militär und Wissenschaft grundsätzlich getrennte Sphären: Militärische Mobilisierung und Kriegserfahrung vermögen zwar dem wissenschaftlichen Fortschritt auf die Sprünge zu helfen, die Inhalte des von Medizin und Wissenschaft produzierten Wissens bleiben aber von der militärischen Welt unberührt.

Diese dominanten Auffassungen zu Wissenschaft und Militär - so viel dürfte der kurze Problemaufriss gezeigt haben - sind ambivalent und widersprüchlich, weisen jedoch insofern Ähnlichkeiten auf, als dass sie Wechselwirkungen zwischen den beiden Systemen negieren. Der in diesem Themenheft angestrebte Versuch, Beziehungen zwischen Wissenschaft und Militär, zwischen Universitätsprofessoren und Generälen, zwischen Industrieforschern und Truppenführern, zwischen Laboratorien und Kasernen historisch in den Blick zu nehmen, erscheint vor diesem Hintergrund als ein irritierendes oder zumindest gewagtes Unterfangen. Die Militärgeschichte sah sich denn auch kaum je veranlasst, einen solchen Versuch zu unternehmen. Eine Ausnahme stellen hier Arbeiten zur Rolle des Militärs in der Kolonialmedizin $^{4}$ oder auch von psychologischer und psychiatrischer Traumaforschung ${ }^{5}$ dar, aber auch diese Arbeiten nahmen selten die Frage auf, inwieweit sich die beiden gesellschaftlichen Teilsysteme gegenseitig beeinflussten. Zwar hat sich die neuere Militärgeschichte längst den Interessen und Methoden der allgemeinen Geschichtsforschung angenähert und sich etwa gegenüber der Sozial-, Wirtschafts-, Kultur- und Geschlechtergeschichte geöffnet. ${ }^{6}$ Es ist jedoch bemerkenswert, dass man auch in neueren programmatischen Veröffentlichungen zur Militärgeschichte vergeblich nach wissenschafts- und wissenshistorischen Perspektiven sucht.?

Im Gegensatz dazu gibt es eine Vielzahl von Studien zu Militär, Krieg und Wissenschaft, die innerhalb der Wissenschafts-, Technik- und Medizingeschichte entstanden sind. Besonders häufig wurden die Rolle von Wissen-

3 Dazu kritisch Eckart 2014, $15 f$.

4 Dieses Forschungsfeld hat in den letzten Jahren grosse Aufmerksamkeit erfahren. Hierzu u.a. Chakrabarti 2014; Surun 2012; Eckart 1997; Zaugg 2016.

5 Quinkert/Rauh/Winkler 2010; Michl/Plamper 2009; Lerner 2003; Lengwiler 2000.

6 Echternkamp 2013.

7 Siehe etwa Müller/Rogg 2013; Vogel/Schmidt/Echternkamp 2010; Kühne/Ziemann 2000. 
schaftlern und von wissenschaftlichen Forschungen in den 'totalen Kriegen' des 20. Jahrhunderts untersucht. ${ }^{8}$ Damit rückten Situationen des Krieges in den Vordergrund und damit ein Extremzustand, der spezifische Voraussetzungen für die Beziehungen von Wissenschaft und Militär schuf. Ein breit untersuchtes Thema stellt etwa die «Selbstmobilisierung»" ${ }^{9}$ von Wissenschaftlern und Gelehrten dar, die als Kriegspropagandisten wirkten, ihre Forschungen in den Dienst von Kriegszielen stellten oder die Kriegsrelevanz ihrer Projekte zu nutzen verstanden, um Ressourcen für ihre Disziplinen, Institute und individuellen Karrieren zu erschließen. ${ }^{10}$ Weitgehend bekannt ist mittlerweile, wie sich etwa Physiker, Ingenieure und Techniker einspannen ließen für die Entwicklung von Kriegstechnologien ${ }^{11}$ oder wie Ärzte bereit waren, ethische Grenzen zu überschreiten, um Kriegskontexte für medizinische Forschungen auszunutzen. ${ }^{12}$ Diese vielfältigen Studien zeigen, wie unzutreffend letztlich die beiden eingangs skizzierten Bilder sind. Militär und Wissenschaft bilden weder Gegensätze noch lassen sich ihre komplexen Beziehungen in simplifizierende Fortschrittsnarrative übersetzen, die den Kosten des Krieges einen angeblichen medizinischen, technischen und wissenschaftlichen Nutzen gegenüberstellen.

Die in diesem Themenheft versammelten Beiträge teilen diese Skepsis und schließen in verschiedener Hinsicht an Frage- und Problemstellungen dieser hier nur kurz umrissenen Untersuchungsfelder der Wissenschafts-, Technik- und Medizingeschichte an. Wir wollen indessen mit diesem Heft dazu anregen, die Gebiete und Perspektiven dieser Forschungen in vier Richtungen zu erweitern. Erstens ist das Militär eine dauerhafte gesellschaftliche Institution, die auch in Friedenszeiten auf Wissen angewiesen ist, sich an der Produktion von Wissen beteiligt und mit Universitäten und anderen Forschungseinrichtungen zusammenarbeitet. Besonders deutlich wird dies in der Schweiz, die seit der Bundesstaatsgründung nie direkt in Kriegshandlungen verwickelt war, in der aber das Milizsystem und die normative Leitfunktion, die das Militär in politisch-gesellschaftlicher Hinsicht lange Zeit ein-

8 Hier seien nur einige wichtige Sammelbände genannt: Berg/Thiel/Walther 2009; Ad Maas/ Hooijmaijers 2009; Mendelsohn/Smith/Weingart 1988. Zum Kalten Krieg siehe Oreskes/ Krige 2014.

9 Der Begriff geht zurück auf Mehrtens 1994.

10 Siehe dazu auch die Forschungsdiskussion in Berg/Thiel/Walther 2009, 10-18.

11 Nur schon zu Deutschland existiert dazu eine Vielzahl von Studien. Siehe etwa: Nagel 2012; Neufeld 2009; Maier 2007; Pulla 2006; Maier 2002.

$12 \mathrm{Zu}$ Menschenversuchen in Kriegskontexten siehe grundlegend: Weindling 2015; Slater 2009; Griesecke/Krause/Pethes/Sabisch 2009. Zur Rolle von Medizin und Ärzten im Ersten und Zweiten Weltkrieg siehe insbesondere: Eckart 2014; Prüll/Rauh 2014; Harrison 2010; Berger 2009; Michl 2007; Eckart/Neumann 2006; Neumann 2005; Eckart/Gradmann 2003; Cooter/Harrison/Sturdy 1999. 
nahm, Kooperationen zwischen Wissenschaft und Militär begünstigten. Wesentlich waren etwa für das Milizsystem typische Rollenkumulationen, so etwa Universitätsprofessoren, die hohe militärische Ränge bekleideten. ${ }^{13}$ Die Autorinnen und Autoren dieser Ausgabe sind bestrebt, solche militärischwissenschaftlichen Verflechtungen zu analysieren und deren Einflüsse auf die Inhalte von Forschungen, auf die Ausgestaltung von Denkstilen sowie auf die Genese und Zirkulation von Wissen herauszuarbeiten.

Zweitens plädieren wir dafür, den Blick nicht zu sehr auf Disziplinen zu verengen, die das Militär - wie etwa die Wehrwissenschaften oder die Atomtechnologie - aufgrund ihres kriegstechnologischen Potentials förderte. So behandeln die Beiträge im Themenheft Wissensfelder, die bislang kaum auf ihre Beziehungen zu militärischen Institutionen, Diskursen und Praktiken befragt wurden, so etwa die Geologie, die Epidemiologie, die Genetik, die Kartographie, die Anthropologie, die Demographie, die Statistik oder das Recht. Zum einen stellte das Militär häufig ein Laboratorium für wissenschaftliche Erkenntnisse dar. So nutzten Wissenschaftler - von Medizinern über Anthropologen bis zu Soziologen - das Militär als Institution, um Untersuchungen durchzuführen oder auf dort gesammelte Daten zurückzugreifen. Zum anderen ist das Militär eine soziale Organisation, in der unterschiedlichste Formen von Wissen angewendet, vermittelt und - etwa in Militärbibliotheken - gespeichert wurden.

Der dritte Punkt betrifft die Verbindung von nationaler und transnationaler Dimension. Wie zentral der grenzüberschreitende Austausch für das Funktionieren von Wissenschaften ist, bedarf hier keiner Ausführungen. Auch für das Militär bildete aber die Nation nicht den einzigen Referenzrahmen. Falsch wäre jedenfalls die Annahme, die Wissenschaft habe einen ausschliesslich transnationalen Bezugsrahmen, während das Militär nur im nationalen Rahmen denke. Ist es der Geschichtswissenschaft nun schon seit langem gelungen, die affirmativen Wechselverhältnisse von «Wissenschaft und Nation» darzustellen, ${ }^{14}$ so sind transnationale Vernetzungen des Militärs - gerade in deren Bedeutung als Ort der Wissensproduktion und -vermittlung - noch wenig erforscht. Militärangehörige beteiligten sich an internationalen Kongressen, begegneten sich bei Manövern oder waren Mitglieder in Fachorganisationen anderer Länder. Die Kommunikations- und Wissenssysteme des Militärs wiesen eine dezidiert transnationale Dimension auf. Die Beiträge in diesem Band beschreiben, wie kriegsrelevantes oder militärisch geprägtes Wissen in unterschiedliche nationale Kontexte diffun- 
dierte, den Atlantik überquerte oder zwischen europäischen und kolonialen Kontexten zirkulierte.

Die drei bisher genannten Punkte deuten bereits den programmatischen Ansatz an, dem sich dieses Themenheft verpflichtet fühlt. Die hier versammelten Aufsätze sehen sich - und damit sei der vierte und übergreifende Punkt genannt - als einen Beitrag zum noch jungen Feld der Wissensgeschichte, die an die Wissenschaftsgeschichte und die Science and Technology Studies der 1980er und 1990er Jahre anknüpft, deren Begrenzungen aber methodisch und thematisch aufzusprengen sucht. ${ }^{15}$ Das gemeinsame Ziel dieses heterogenen und noch kaum kanonisierten Feldes besteht darin, Entstehung, Ausbreitung, Stabilisierung und Zerfall von Wissen historisch zu erforschen. Wissen wird dabei als ein wandelbares und mobiles Objekt untersucht, das wissenschaftliche, geographische und institutionelle Grenzen überschreitet, zwischen akademischen und populären Wissenskulturen zirkuliert, unterschiedliche mediale Formate annimmt, als Ressource in diversen sozialen, ökonomischen und politischen Kontexten dient und dabei stets nicht nur den Ort wechselt, sondern auch umgestaltet wird. Im Unterschied zur klassischen Wissenschaftsgeschichte beschränkt sich die Wissensgeschichte deshalb nicht auf einzelne wissenschaftliche Disziplinen, sondern richtet ihren forschenden Blick auf die Produktion und Zirkulation von Wissen in breiten politischen und gesellschaftlichen Zusammenhängen. Sie zielt insofern, wie etwa die Historiker David Gugerli und Daniel Speich Chassé betonen, auf die Untersuchung «breiter soziopolitischer Konstellationen» ab und will mithin auch «Machtverhältnisse» explizit zu ihrem Gegenstand machen. ${ }^{16}$

Vor diesem Hintergrund ist es erstaunlich, dass das Militär, das wie keine andere Institution in der Moderne Macht und Gewalt ausübte und zu diesem Zweck auf Wissen angewiesen war, im neuen Feld der Wissensgeschichte noch wenig Beachtung gefunden hat. Unseres Erachtens stellt das Militär ein geeignetes Untersuchungsobjekt dar, um Fragen nach Machtverhältnissen, Exklusionspraktiken und Hierarchien in die Untersuchung von Wissensformationen zu integrieren. Hierarchische Autoritätsstrukturen, die Gliederungen der bürgerlichen Gesellschaften wiederspiegeln; eine Beschränkung auf die 'leistungsfähigen' Bürger des Landes, die verschiedenen Selektionskriterien genügen müssen; und nicht zuletzt die - zumindest in den hier untersuchten Zeiträumen - vollständige Beschränkung auf männliche Staatsbürger, die zum Sinnbild der gesamten Gesellschaft stilisiert wurden: Alle diese Elemente kennzeichneten das Militär auch in seiner Bedeutung als Ort der

15 Siehe dazu programmatisch: Gugerli/Speich Chassé 2012; Sarasin 2011; Vogel 2004. 16 Gugerli/Speich Chassé 2012, 93. 
Wissensproduktion. Anhand des Militärs lässt sich herausarbeiten, wie gesellschaftliche Machtasymmetrien und Hierarchien ihren Niederschlag in den Wissensordnungen moderner Gesellschaften fanden. Wir wollen das Militär deshalb als einen Ort der Wissensgewinnung, -vermittlung und -anwendung in den Blick nehmen und dabei untersuchen, wie die Organisationsformen, Denkstile und Zielsetzungen dieser Institution dieses Wissen beeinflussten. Inwiefern etwa prägten militärische Hierarchien und Machtverhältnisse das im Militär produzierte und verwendete Wissen? Welche Rolle spielten Geschlechterordnungen bei der Ausgestaltung militärischer Wissensfelder- und -systeme? Welche Wechselwirkungen bestanden zwischen akademisch geprägten und militärisch dominierten Wissensfeldern? Wie zirkulierte Wissen zwischen zivilen und militärischen Institutionen, und wie veränderten diese Übersetzungsprozesse das Wissen?

Die Autorinnen und Autoren der folgenden Beiträge nähern sich diesen Fragen aus unterschiedlichen Perspektiven und arbeiten eine Vielzahl von Querbeziehungen zwischen den vermeintlich klar getrennten Sphären von Militär und Wissenschaft heraus. Dabei wird nicht nur der Einfluss des Militärs etwa in den Bereichen von Kartografie und Geologie (Thoral und Hartmann) ersichtlich, sondern auch das spezifische Potential des Militärs als statistisch und demographisch untersuchbares «Reservoir von Vitaldaten» (Fredj, Goltermann, Germann). Hier wird deutlich, dass das Militär nicht nur eine institutionelle Plattform für neue Wissensdiskurse bot. Vielmehr prägte es auch eine neue Kultur statistischer und zahlenbasierter Wahrheitsansprüche und Evidenzkonstruktionen wesentlich mit. Die Studien greifen Fälle zwischen dem frühen 19. Jahrhundert und der Mitte des 20. Jahrhunderts auf und setzen dabei unterschiedliche geographische Schwerpunkte. Hartmann und Germann nehmen das spezifische Verhältnis von Militär und Gesellschaft in der Schweiz in den Blick, während Thoral und Fredj mit dem zentralistischen und imperialen Frankreich auf denjenigen Nationalstaat fokussieren, der über die älteste europäische Wehrpflichtarmee verfügte. Goltermanns Beitrag weitet den geographischen Fokus aus und untersucht Zirkulationen von Wissen in einem gesamteuropäischen Kontext. Die hier behandelten Beispiele und Themen verfolgen indessen keineswegs den Anspruch, ein umfassendes Bild des Austausches zwischen Wissenschaft und Militär in Europa zu vermitteln. Vielmehr handelt es sich um Sondierungen, welche die Möglichkeiten der oben skizierten wissensgeschichtlichen Perspektiven auf das Militär ausloten.

Svenja Goltermann untersucht in ihrem Beitrag die Rolle, die medizinisches und juristisches Wissen in den Konzepten eines gerechten Krieges im 
langen 19. Jahrhundert gespielt haben. Sie geht dabei von der genuinen Bedeutung aus, die Prozesse wie Zählen und Beobachten, Fotografieren und Untersuchen im Selbstverständnis der auf Zukunftswissen ausgelegten Gesellschaft gespielt haben. Das Militär wurde hier, gerade auch in Kriegssituationen, zu einem Observatorium und Experimentierfeld, auf die sich Vorstellungen von einer gerechten Zukunft des nationalstaatlich verstandenen Europas stützten. Der Beitrag zeigt, welche ambivalenten Effekte medizinisches Wissen, das Ärzte im Militär und im Krieg gewannen, für die Entwicklung humanitärer und völkerrechtlicher Ideen zeitigte: Es konnte zur Linderung von Leid und zu Verboten bestimmter Waffen beitragen, aber es wurde auch für die Entwicklung von Waffentechniken und zur Legitimation von Kriegen eingesetzt.

In ihrem Beitrag zum Aufbau von Militärbibliotheken in der französischen Armee im Gefolge der napoleonischen Kriege weist Marie-Cécile Thoral darauf hin, dass die Schaffung spezieller geographischer Sammlungen und Bibliotheken seit dem frühen 19. Jahrhundert wesentlich von ihrer strategischen Bedeutung für das Militär abhing. Die spezifische Institution der militärischen Sammlungen erlaubte erst die Kompilation eines relevanten Fachwissens, das häufig zunächst als Arkanwissen behandelt wurde. Nur der exklusive Zugang über das Militär ermöglichte es somit, diese Wissensbestände zu ordnen und in Kartenform für neue Zwecke nutzbar zu machen. Das so erarbeitete kartographische und geographische Wissen fand Eingang in die militärische Ausbildung, die durch den Aufbau der militärischen Sammlungen auch institutionell beeinflusst wurde.

Die Konstituierung eines neuen Raumwissens steht auch im Mittelpunkt des Aufsatzes von Claire Fredj. Gerade in Phasen der kolonialen Expansion europäischer Mächte spielte das Militär in der Entwicklung von Raumrepräsentationen und -kenntnissen eine wesentliche Rolle. Anhand einer Fallstudie zu Algerien in der Mitte des 19. Jahrhunderts zeigt der Aufsatz, wie im Laufe der französischen Militärexpansion neue Krankheitsbilder entdeckt wurden. Die beteiligten Militärmediziner betrachteten die entdeckten Krankheiten nicht nur als medizinische Herausforderungen, sondern versuchten, auf der Basis dieses medizinischen Wissens neue Raumordnungen zu formulieren. Dies verdeutlicht besonders die Produktion statistischen Wissens über die Risiken, welchen europäische Truppen im subtropischen und tropischen Klima ausgesetzt waren. Dieses Wissen diente mitunter als Reservoir, aus welchem sich Argumente kolonialer Dominanz speisen ließen.

Überschneidungen von geographischem und statistischem Wissen und verschiedenen demographischen Diskursen stehen auch im Fokus des Beitrags 
von Heinrich Hartmann. Anhand der Untersuchungen zum Vorkommen des Kropfes und der «cretinischen Degeneration» in der Schweiz zeigt der Beitrag, dass die Wahrnehmung der Krankheiten als eine Bedrohung der nationalen Wehrkraft für viele Wissenschaftler die Möglichkeit bot, einerseits ihr Wissen zu vermitteln, andererseits aber statistische Untersuchungen durchzuführen. Am Beispiel des Aargauer Militärarztes Heinrich Bircher wird deutlich, dass die hygienischen Präventionsbestrebungen auch mit einem neuen eugenischen Wissensdiskurs konvergierten, zumal sich Birchers statistische Extrapolationen für rassenbiologische Interpretationen verwenden ließen.

In seinem Aufsatz zur Sammlung von Blutkonserven und Blutgruppendaten in der Schweiz während des Zweiten Weltkriegs und im frühen Kalten Krieg arbeitet Pascal Germann heraus, wie stark der Aufbau eines medizinischen Blutspendedienstes durch militärische Interessen und Akteure geprägt war. Von dieser «Mobilisierung des Blutes» profitierten Wissenschaftler, die unter anderem Zugang zu umfangreichen militärischen Daten und zu neu aufgebauten serologischen Laboratorien erhielten. Während sich für die Wissenschaft neue Möglichkeiten der humangenetischen, medizinischen und anthropologischen Forschung auftaten, verfolgten Militärs mit einem effizienten Bluttransfusionsdienst das Ziel einer permanenten Kriegsbereitschaft. Der Aufsatz zeigt, wie es in der Schweiz des frühen Kalten Krieges zu vielfältigen Kooperationen zwischen Militär, Medizin und Wissenschaft kam.

Die Beiträge illustrieren die Vielzahl von Forschungsfragen, welche die komplexen Wechselverhältnisse von Wissenschaft und Militär sowie die vielfältigen Beziehungen des Militärs zur gesellschaftlichen Genese und Transformation von Wissen aufwerfen. Es wird weiter zu diskutieren und zu erforschen sein, ob das Militär die Entwicklungen zur modernen Wissensgesellschaft befördert oder ob sich die hierarchischen Strukturen und die auf den Krieg ausgerichteten Ziele des Militärs eher als Hemmschuh einer solchen Entwicklung ausgewirkt haben. Die in diesem Themenheft versammelten Anhaltspunkte, Thesen und Perspektiven sollen deshalb nicht zuletzt zu weiteren Forschungen zur Wissensgeschichte des Militärs anregen.

\section{Bibliographie}

Ad Maas/Hans Hooijmaijers (eds.), Scientific Research in World War II: What Scientists Did in the War (London, New York 2009)

Berg, Matthias/Jens Thiel/Peter Th. Walther (Hrsg.), Mit Feder und Schwert. Militär und Wissenschaft - Wissenschaftler und Krieg (Stuttgart 2009) 
Berger, Silvia, Bakterien in Krieg und Frieden. Eine Geschichte der medizinischen Bakteriologie in Deutschland, 1890-1933 (Göttingen 2009)

Chakrabarti, Pratik, Medicine and Empire 1600-1960 (Basingstoke 2014)

Cooter, Roger/Mark Harrison/Steve Sturdy (eds.), Medicine and Modern Warfare (Amsterdam 1999)

Diner, Dan, Das Jahrhundert verstehen. Eine universalhistorische Deutung (Frankfurt a.M. 2000)

Echternkamp, Jörg, «Militärgeschichte, Version: 1.0», in: Docupedia-Zeitgeschichte (12.07.2013)

Eckart, Wolfgang U., Medizin und Krieg: Deutschland 1914-1924 (Paderborn 2014)

Eckart, Wolfgang U., Medizin und Kolonialimperialismus: Deutschland 1884-1945 (Paderborn 1997)

Eckart, Wolfgang U./Alexander Neumann (Hrsg.), Medizin im Zweiten Weltkrieg: Militärmedizinische Praxis und medizinische Wissenschaft im «Totalen Krieg» (Paderborn/Zürich 2006)

Eckart, Wolfgang U./Christoph Gradmann (Hrsg.), Die Medizin und der Erste Weltkrieg (Herbolzheim 2003)

Griesecke, Birgit/Marcus Krause/Nicolas Pethes/Katja Sabisch (Hrsg.), Kulturgeschichte des Menschenversuchs im 20. Jahrhundert (Frankfurt a.M. 2009)

Gugerli, David/Daniel Speich Chassé, «Wissensgeschichte. Eine Standortbestimmung», in: Traverse 1 (2012), 85-100

Hagner, Michael (Hrsg.), Wissenschaft und Demokratie (Berlin 2012)

Harrison, Marc, The Medical War: British Military Medicine in the First World War (Oxford 2010)

Kühne, Thomas/Benjamin Ziemann (Hrsg.), Was ist Militärgeschichte? (Paderborn, München, Wien, Zürich 2000)

Lengwiler, Martin, Zwischen Klinik und Kaserne. Die Geschichte der Militärpsychatrie in Deutschland und der Schweiz 1870-1914 (Zürich 2000)

Lerner, Paul, Hysterical Men: War, Psychiatry, and the Politics of Trauma in Germany, 1890-1930 (Ithaca 2003)

Livia Prüll/Philipp Rauh (Hrsg.), Krieg und medikale Kultur. Patientenschicksale und ärztliches Handeln in der Zeit der Weltkriege 1914-1945 (Göttingen 2014)

Maier Helmut (Hrsg.), Rüstungsforschung im Nationalsozialismus. Organisation, Mobilisierung und Entgrenzung der Technikwissenschaften (Göttingen 2002)

Maier, Helmut, Forschung als Waffe. Rüstungsforschung in der Kaiser-WilhelmGesellschaft und das Kaiser-Wilhelm-Institut für Metallforschung, 1900-1945/48 (Göttingen 2007)

Mehrtens, Herbert, «Kollaborationsverhältnisse. Natur- und Technikwissenschaften im NS-Staat und ihre Historie», in: Meinel, Christoph/Peter Voswinckel (Hrsg.), Medizin, Naturwissenschaft, Technik und Nationalsozialismus. Kontinuitäten und Diskontinuitäten (Stuttgart 1996) 13-32

Mendelsohn, Everett/Merrit Roe Smith/Peter Weingart (eds.), Science, Technology, and the Military, Vol. 1 and 2 (Dordrecht 1988)

Michl, Susanne, Im Dienste des «Volkskörpers». Deutsche und französische Ärzte im Ersten Weltkrieg (Göttingen 2007) 
Michl, Susanne/Jan Plamper, «Soldatische Angst im Ersten Weltkrieg: Die Karriere eines Gefühls in der Kriegspsychiatrie Deutschlands, Frankreichs und Russlands», Geschichte und Gesellschaft 35/2 (2009) 209-248

Müller, Christian Th./Matthias Rogg (Hrsg.), Das ist Militärgeschichte! Probleme, Projekte, Perspektiven (Paderborn 2013)

Nagel, Günther, Wissenschaft für den Krieg. Die geheimen Arbeiten der Abteilung Forschung des Heereswaffenamtes (Stuttgart 2012)

Neufeld, Michael J., Wernher von Braun. Visionär des Weltraums, Ingenieur des Krieges (München 2009)

Neumann, Alexander, «Arzttum ist immer Kämpfertum». Die Heeressanitätsinspektion und das Amt «Chef des Wehrmachtssanitätswesens» im Zweiten Weltkrieg (1939-1945) (Düsseldorf 2005)

Oreskes, Naomi/John Krige (eds.), Science and Technology in the Global Cold War (Cambridge, Massachusetts 2014)

Pulla, Ralf, Raketentechnik in Deutschland. Ein Netzwerk aus Militär, Industrie und Hochschulen 1930 bis 1945 (Frankfurt a.M. 2006)

Quinkert, Babette/Philipp Rauh/Ulrike Winkler (Hrsg.), Krieg und Psychatrie, 1914-1950 (Göttingen 2010)

Sarasin, Philipp, «Was ist Wissensgeschichte?» Internationales Archiv für Sozialgeschichte der Deutschen Literatur 36/1 (2011), 159-172

Slater, Leo Barney, War and Disease: Biomedical Research on Malaria in the Twentieth Century (New Brunswick 2009)

Surun, Isabelle (dir.), Les sociétés coloniales à l'âge des empires, 1850-1960 (Neuillysur-Seine 2012)

Tanner, Jakob, «Militär und Gesellschaft in der Schweiz nach 1945», in: Ute Frevert (ed.), Militär und Gesellschaft im 19. und 20. Jahrhundert (Stuttgart 1997) 314-341

Vogel, Jakob, «Von der Wissenschafts- zur Wissensgeschichte. Für eine Historisierung der 'Wissensgesellschaft'», Geschichte und Gesellschaft 30 (2004) 639-660

Vogel, Jakob/Ralph Jessen (Hrsg.), Wissenschaft und Nation in der europäischen Geschichte (Frankfurt a. M. 2002)

Vogel, Thomas/Wolfgang Schmidt/Jörg Echternkamp (Hrsg.), Perspektiven der Militärgeschichte: Raum, Gewalt und Repräsentation in historischer Forschung und Bildung (München 2010)

Weindling, Paul, Victims and Survivors of Nazi Human Experiments: Science and Suffering in the Holocaust (London 2015)

Zaugg, Roberto (Hrsg.), Themennummer «Guerres, maladies et empires. Les services de santé militaires en situation coloniale pendant le long $\mathrm{XIX}^{\mathrm{e}}$ siècle», Histoire, médecine et santé, 5 (2016), in Vorbereitung 